\title{
DOSSIER
}

\section{LOS USOS DE LA INVESTIGACIÓN-ACCIÓN-PARTICIPATIVA (IAP) PARA EL ESTUDIO DE LOS RIESGOS PSICOSOCIALES EN EL TRABAJO. REFLEXIONES A PARTIR DE UNA EXPERIENCIA CON REPRESENTANTES SINDICALES Y TRABAJADORES}

THE USES OF PARTICIPATORY ACTION RESEARCH IN THE STUDY OF PSYCHOSOCIAL RISKS AT WORK. REFLECTIONS FROM AN EXPERIIENCE WITH TRADE UNIONISTS AND WORKERS

María Laura Henry²

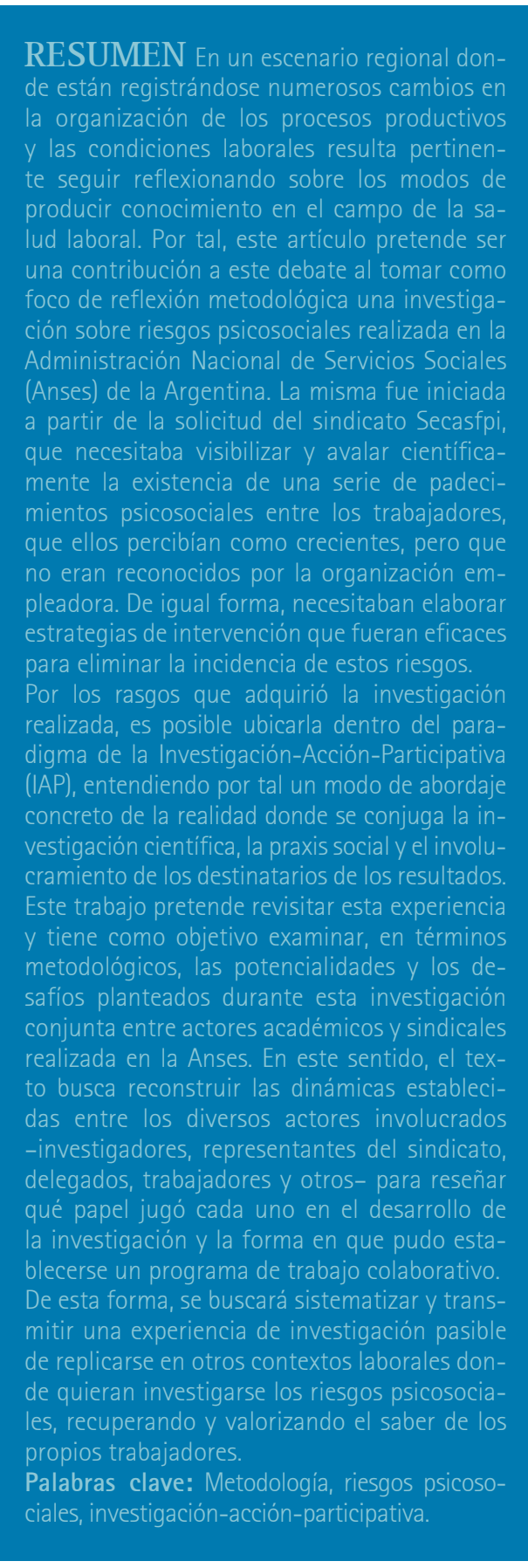

D01: http://dx.doi.org/10.30972/dpd.792803

'Artículo Recibido: 11 de Septiembre 2017 - Aceptado: 1 de noviembre 2017

\section{INTRODUCCIÓN}

Los procesos productivos y las estrategias de gestión de la fuerza de trabajo incesantemente se renuevan en un contexto donde las organizaciones -tanto públicas como privadas- otorgan cada vez más énfasis a la productividad, la eficiencia y la modernización de sus funciones. Innegablemente, esto conlleva repercusiones sobre las condiciones laborales y, por ende, puede afectar la salud de los trabajadores de maneras imprevistas y poco conocidas. Por ello, es conveniente seguir indagando sobre los riesgos -tradicionales y novedosos- a los que están expuestos los trabajadores en el contexto productivo actual.

Teniendo como horizonte estas preocupaciones, el equipo interdisciplinario al que pertenecemos realizó una investigación sobre la incidencia de los riesgos psicosociales (RPST) para el caso de los trabajadores de la Anses (Administración Nacional de Servicios Sociales) de la Argentina, a partir de la solicitud del sindicato Secasfpi ${ }^{3}$ que los representa.

El acercamiento del sindicato al equipo de investigadores surgió por la necesidad del primero de visibilizar y avalar científicamente la existencia de una serie de padecimientos psicosociales entre los trabajadores, que ellos percibian como crecientes, pero que no eran reconocidos por la organización empleadora. Debido a esta invisibilización y a la falta de atención, el sindicato comenzó a buscar articulaciones con el mundo académico para generar datos científicamente probados, que pudiesen impulsar una política preventiva y reparadora integral para los RPST.

Durante el desarrollo de toda la investigación, los miembros del Secasfpi tuvieron una participación activa y un genuino involucramiento, generándose así permanentes instancias de intercambio con el equipo de investigación. Fue así como fue configurándose un trabajo conjunto entre ambas partes, donde dialogaron saberes diversos y confluyeron distintos posicionamientos respecto del campo bajo análisis.

Este artículo pretende revisitar esta experiencia de investigación-acción y se propone como objetivo examinar, en términos metodológicos, las potencialidades y los desafíos planteados 
De Prácticas y discursos

Universidad Nacional del Nordeste

Centro de Estudios Sociales

Año 7, Número 9, 2018, Marzo

ISSN 2250-6942

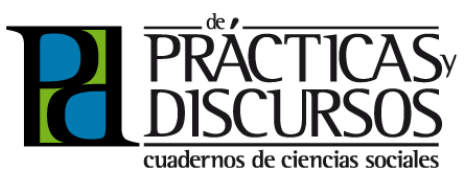

durante esta investigación conjunta entre actores académicos y sindicales realizada en la Anses. En este sentido, el texto busca reconstruir las dinámicas establecidas entre los diversos actores involucrados -investigadores, representantes del sindicato, delegados, trabajadores y otros- para reseñar qué papel jugó cada uno en el desarrollo de la investigación y la forma en que pudo establecerse un programa de trabajo colaborativo. Asimismo, en términos más generales, este artículo pretende ser un aporte para repensar las actuales formas en que trabajadores y expertos abordan la problemática de los riesgos laborales, proponiendo un diseño alternativo de investigación que puede ser replicado en otros contextos laborales.

Por los rasgos que adquirió la investigación realizada, es posible ubicarla dentro de lo que se denomina el paradigma de la Investigación-Acción-Participativa (IAP), entendiendo por tal un modo de abordaje concreto de la realidad donde se conjuga la investigación científica, la praxis social y el involucramiento de los destinatarios de los resultados. De esta forma, comenzaremos reseñando los núcleos más significativos de la IAP, para luego mostrar su

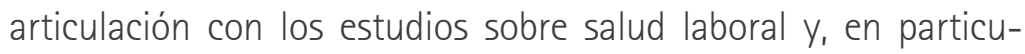
lar, con el enfoque de los riesgos psicosociales en el trabajo. A continuación, nos detendremos a analizar tres aspectos donde se generó una estrecha participación entre los investigadores y los destinatarios del proyecto: la composición del grupo de trabajo, el desarrollo del trabajo de campo, y la elaboración del diagnóstico y de las propuestas de intervención. Por último, ofreceremos unas reflexiones a modo de conclusión y balance general.

\section{LOS PRINCIPIOS DE LA}

INVESTIGACIÓN-ACCIÓN-PARTICIPATIVA (IAP)

Y SUS USOS EN LOS ESTUDIOS DE SALUD LABORAL

\subsection{PRINCIPALES CARACTERISTICAS DE LA IAP}

Como tarea preliminar, resulta fructífera una reflexión de tipo conceptual referida al tipo de investigación que estamos examinando. Al respecto, Ander-Egg (2002) define a la IAP como un proceso que supone la simultaneidad de conocer y de intervenir, e implica la participación de la misma gente involucrada en el programa de estudio y de acción. Por su parte, Park (1992) indica 
De Prácticas y discursos

Universidad Nacional del Nordeste

Centro de Estudios Sociales

Año 7, Número 9, 2018, Marzo

ISSN 2250-6942
Los usos de la investigación-acción-participativa [IAP] para el estudio de los riesgos psicosociales en el trabajo. Reflexiones a partir de una experiencia con representantes sindicales y trabajadores

The uses of participatory action research in the study of psychosocial risks at work. Reflections from an experience with trade unionists and workers

que la IAP comparte con la ciencia social tradicional el uso de sus métodos, pero se diferencia por la especificidad de los objetivos - de cambio social- que persigue, la clase de conocimiento que genera y por la manera de relacionar el conocimiento con la acción social. Park, además, enfatiza que la IAP opta por trabajar con aquellos grupos sociales que tienen escaso poder y recursos de forma tal que, a través de este tipo de investigaciones, puedan adquirir conocimientos y capacidades que les permitan mejorar su situación de forma autónoma. Finalmente, Campos (1990) profundiza en las premisas y raíces históricas de la IAP y señala que esta puede ser entendida como la síntesis crítica de los avances tercermundistas en materia de investigación-acción, y que sus fundamentos epistemológicos provienen, en lo esencial, de la dialéctica materialista. En este sentido, indica el autor, constituye una forma de investigación social que aspira a la articulación crítica de dos formas de producción del conocimiento que se han visto históricamente escindidas, hasta el punto de situarse a menudo como alternativas antagónicas: la investigación científica social y el conocimiento popular.

Más allá de la variedad de definiciones existentes, todas comparten que la IAP contiene una serie de elementos constitutivos que le brindan su identidad y especificidad:

a) El uso del método científico y la definición de los objetivos por los destinatarios

La IAP constituye un procedimiento reflexivo, sistemático y controlado que tiene por finalidad estudiar algún aspecto de la realidad. De esta forma, se usan muchas de las técnicas y diseños provenientes de la investigación tradicional pero, en este caso, con una expresa finalidad práctica. En lo referido al objetivo de la investigación -determinar lo que se va a estudiar-, el mismo se decide a partir de lo que interesa a un grupo o colectivo social. No se trata de estudiar problemas de interés científico o cuestiones que preocupan a un grupo de investigadores, sino aquellos problemas que las personas involucradas consideran importantes porque tienen que ver con aspectos que conciernen a sus propias vidas (Ander-Egg, 2002).

b) Orientación hacia la transformación de la realidad

La IAP procura establecer una dialéctica entre el conocimiento y la acción: no solo se trata de conocer la realidad, sino de actuar sobre ella. La finalidad última del estudio es la transformación de la situación-problema que afecta a la gente involucrada (Ander-Egg, 
De Prácticas y discursos

Universidad Nacional del Nordeste

Centro de Estudios Sociales

Año 7, Número 9, 2018, Marzo

ISSN 2250-6942

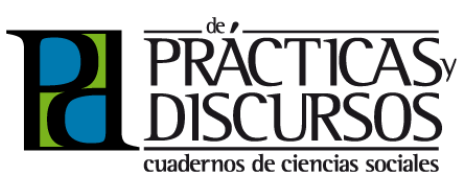

\begin{abstract}
${ }^{4}$ El problema es que, por su propia historia de subordinación o explotación, a los miembros de estos grupos no les resulta sencillo tomar la iniciativa para transformar su realidad, y en esto los investigadores externos juegan un papel crítico en tanto catalizadores. Deben ayudarlos para que desarrollen una conciencia crítica de la realidad y realicen su potencial trasformador (Balcazar, 2003).
\end{abstract}

2002). En concordancia con esta orientación, la IAP no sostiene la neutralidad valorativa del proceso de investigación. Por el contrario, considera el importante papel que tiene el científico social en disminuir la injusticia en la sociedad, promover la participación de los miembros de las comunidades en la búsqueda de soluciones a sus problemas y ayudarlos a incrementar el grado de control que ellos tienen sobre aspectos relevantes de sus vidas (Balcazar, 2003).

\section{c) Conocimiento interactivo}

La relación de los actores involucrados en la IAP no es la de sujeto-objeto, como en la investigación clásica, sino la de sujeto-sujeto. Así, de entidad que se presupone pasiva y externa, la gente deviene sujeto protagonista de todo un proceso de trasformación de su realidad (Ander-Egg, 2002).

Asimismo, esta relación presupone un intercambio entre ambas partes, donde los científicos sociales proveen sus habilidades de sistematización, teorias para interpretar la realidad y métodos de recolección de datos, mientras que los destinatarios proveen su conocimiento social y un acervo de saberes -con su especificidad y atributos que no lo hacen inferior ni superior a la ciencia, sino diferente. Ambos se relacionan de modo dialógico y permiten encarar el proyecto común de transformación social.

En suma, el rol de los investigadores es de ayudar a sistematizar las experiencias populares y devolverlas a la misma gente ${ }^{4}$. Esta restitución sistemática y sistematizada de saberes aporta nuevos conocimientos a los sectores populares y suscita nuevas perspectivas para lograr una lectura más crítica de su realidad (Ander-Egg, 2002).

d) Empoderamiento de los grupos sociales involucrados Respecto de este punto, Balcazar (2003) señala que durante la IAP los participantes aprenden a desarrollar una conciencia crítica que les permite identificar las causas de sus problemas -alejándolos de posiciones victimizantes y de la desesperanza aprendida- e identificar posibles soluciones. El propósito es enseñar a la gente a descubrir su propio potencial para actuar, liberándolos de estados de dependencia y pasividad previos. De igual forma, Park (1992) explica que la IAP actúa como una intervención catalítica en los procesos de transformación, apoyando a los grupos con poco poder y escasos medios para que puedan cambiar facetas de su medio social en pos de una vida más plena. Así, la finalidad de este tipo de investigaciones consiste en apoyarlos para que sean autónomos, confien en sí mismos y lleguen a la autodeterminación. El conjunto de los rasgos reseñados le otorgan a la IAP su pecu- 
De Prácticas y discursos

Universidad Nacional del Nordeste

Centro de Estudios Sociales

Año 7, Número 9, 2018, Marzo

ISSN 2250-6942
Los usos de la investigación-acción-participativa [IAP] para el estudio de los riesgos psicosociales en el trabajo. Reflexiones a partir de una experiencia con representantes sindicales y trabajadores

The uses of participatory action research in the study of psychosocial risks at work. Reflections from an experience with trade unionists and workers

liaridad y también dejan en evidencia que no existe pues, como algunos afirman, una oposición metodológica entre la IAP y los procedimientos clásicos de investigación social. La IAP recurre a dichos procedimientos, pero lo hace de una manera flexibilizada y con ciertas peculiaridades que surgen por la activa participación de la gente y por los fines de transformación que persigue.

\subsection{LA APLICACIÓN DE LA IAP EN EL ESTUDIO DE LA SALUD Y LOS RIESGOS LABORALES}

Al igual que en muchas otras esferas, la IAP ha tenido una valiosa aplicación en el campo de la salud laboral y se desprenden de ella grandes posibilidades. Como señala Martínez Alcántara (2007), concebir a los trabajadores como agentes idóneos para emitir opiniones y con capacidad de decisión sitúa a la IAP como una acción de enorme potencial político, orientada a transformar la realidad en función de un beneficio colectivo. No es casual, señala la autora, que la investigación participativa haya tenido su auge en las década de 1960 y 1970, es decir, en un periodo de fuertes críticas a los paradigmas establecidos, de movimientos sociales que luchaban por salir de la subordinación y en el que se desarrollaron riquisimas experiencias que hasta nuestros dias siguen siendo una referencia para este tipo de investigaciones.

Fue justamente en esa época que en Italia se desarrolló una de las experiencias más ejemplares para quienes estudian la salud y los riesgos en el trabajo: el denominado Modelo Obrero Italiano. Surgido a mediados de los sesenta en el norte industrial de ese país, en el marco de una revitalización del movimiento sindical de tipo clasista y de la difusión de novedosas formas de participación de base (consejos obreros), este modelo representó una visión diferente de hacer investigación sobre salud en el trabajo, dado que la participación de los trabajadores constituía el punto de partida. Este modelo reconceptualizó en términos metodológicos, y sobre todo políticos, la concepción que primaba sobre la salud laboral, señala Laurell (1984). Hasta ese momento se había naturalizado, por una parte, que la salud era una especie de mercancía con un precio asociado a ella, por lo cual la "solución" frente a los daños generados por el trabajo era ofrecer una compensación monetaria al trabajador; por otra parte, se concebia que el proceso productivo -con sus efectos nocivos sobre la salud-obedecía a la lógica impersonal y neutral del progreso científico técnico y, 
De Prácticas y discursos

Universidad Nacional del Nordeste

Centro de Estudios Sociales

Año 7, Número 9, 2018, Marzo

ISSN 2250-6942

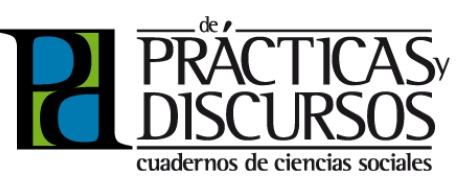

\footnotetext{
${ }^{5}$ Este mismo mapa de riesgos permitía posteriormente a los trabajadores monitorear las medidas que se iban aplicando para contrarrestar los riesgos, así como controlar su efectividad a lo largo del tiempo.
}

por tanto, resultaba esencialmente inmodificable.

Como explica Laurell (1984), la consigna levantada por el movimiento obrero italiano de "la salud no se vende" marcó no solo un nuevo campo de lucha, sino también un cambio de concepción profundo sobre lo que estaba en juego. En primer término, el Modelo Obrero sacó la cuestión de la salud de la lógica mercantil, por cuanto la ubicó no como una parte cotizable de la fuerza de trabajo, sino como parte vital del obrero. Dejó de ser mercancía y devino en potencialidad humana, en algo irrenunciable. En segundo término, significó una rebelión contra la idea de lo inmutable de la organización capitalista del proceso de trabajo. La demanda obrera no podia reducirse a indemnizaciones por daño, sino que debía cuestionar y transformar aquellas condiciones de trabajo que desgastaban y enfermaban a los obreros.

A modo de síntesis, es posible enunciar algunos de los principios básicos que vertebraban al Modelo Obrero (Martínez Alcántara, 2007; Laurell, 1984; Vogel, 2016):

-La no delegación de la salud: los trabajadores deben apropiarse de ella de manera responsable, sin delegarla a la empresa o a consultores contratados por esta (médicos, ingenieros, técnicos en seguridad, etc.).

-El reconocimiento de las capacidades de los propios trabajadores para evaluar sus condiciones de trabajo. En términos prácticos, para esta evaluación en el Modelo Obrero se procedia a la conformación de grupos homogéneos de exposición que participan en la aplicación de una encuesta sobre condiciones de trabajo y de salud, para identificar -y luego debatirconsensuar- los principales problemas y riesgos laborales al interior del centro laboral.

-Elaboración de una plataforma de demandas: con la información recolectada en la encuesta y el acuerdo alcanzado sobre los principales problemas, se realizaban dos acciones posteriores. Por un lado, la confección de un mapa de riesgo, que es la representación visual del proceso laboral con sus riesgos y daños a la salud ${ }^{5}$. Por otra parte, sobre la base del conocimiento así generado, la elaboración de una plataforma de demandas, de nuevo a través de un procedimiento de validación consensual, y la demarcación de una estrategia de lucha para lograrlas.

¿Sirve en la actualidad el Modelo Obrero? ¿Es viable su aplicación en el escenario actual? Son preguntas muy pertinentes, dado que se desarrolló en un contexto histórico donde el "empleo típico" 
De Prácticas y discursos

Universidad Nacional del Nordeste

Centro de Estudios Sociales

Año 7, Número 9, 2018, Marzo

ISSN 2250-6942
Los usos de la investigación-acción-participativa (IAP) para el estudio de los riesgos psicosociales en el trabajo. Reflexiones a partir de una experiencia con representantes sindicales y trabajadores

The uses of participatory action research in the study of psychosocial risks at work. Reflections from an experience with trade unionists and workers

era mayoritario y poseía una serie de rasgos comunes: lugar de trabajo estable, un perfil de trabajadores bastante homogéneo (hombres, no extranjeros, de cuello azul) y unas relaciones de contratación formales por tiempo indeterminado, señala Martínez Alcántara (2007). Pero todo esto ha cambiado y la desregulación se ha impuesto como la norma en las últimas décadas, haciendo que el trabajo ya no sea fijo, estable, presencial, a tiempo completo. La alternancia de empleos, la precariedad, la incertidumbre y la diversidad al interior de los colectivos de trabajadores constituye hoy la experiencia predominante en muchos sectores económicos.

Ciertamente esto representa importantes desafíos pero, como señala la autora, siguen siendo válidos los principios que vertebran al Modelo Obrero: la recuperación de la experiencia obrera y la no delegación de la salud. Ahí reside el potencial transformador de este enfoque y el legado para modelos alternativos actuales, donde los trabajadores sean protagonistas de su historia, con capacidad de decidir de qué no quieren enfermar y de qué no quieren morir (Martínez Alcántara, 2007).

Como veremos a continuación, enfoques actuales como el de los riesgos psicosociales se nutren de esta forma de concebir la salud en el trabajo y la incorporan dentro de un entramado conceptual moderno, adaptado a la realidad laboral presente, de forma tal que se logra un potencial de comprensión y de acción más abarcador.

\subsection{EL ENFOQUE DE LOS RIESGOS PSICOSOCIALES}

Tradicionalmente, los enfoques que abordan la salud laboral han puesto el énfasis en los aspectos físicos del trabajador, en los riesgos directamente visibles (químicos, biológicos, físicos) y en un acotado número de enfermedades y accidentes. Esta perspectiva se volvió hegemónica en un contexto donde el trabajo era eminentemente industrial, físico y referido a empleos típicos. Pero en los últimos años, los análisis han empezado a tomar en consideración que las facetas psicosociales del trabajador también pueden verse afectadas por el trabajo en determinadas circunstancias.

Como explica Neffa (2015), los riesgos psicosociales se intensificaron y se visibilizaron cuando se instauró un nuevo régimen de acumulación capitalista a mediados de la década de 1970 -para enfrentar la crisis surgida en esos años- y que se encuentra vigente hasta hoy. Este nuevo régimen tiene, entre otras, una serie 
De Prácticas y discursos

Universidad Nacional del Nordeste

Centro de Estudios Sociales

Año 7, Número 9, 2018, Marzo

ISSN 2250-6942

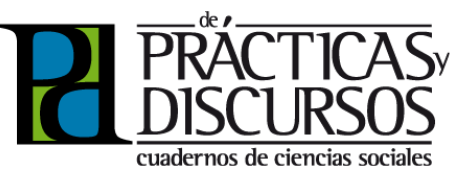

de caracteristicas que impactan fuertemente sobre los trabajadores: la intensificación del trabajo, la desregulación de los vínculos laborales, la flexibilización laboral y, fundamentalmente, la creación de valor a partir del uso de las habilidades psíquicas, emocionales y cognitivas de los trabajadores.

En este marco, padecimientos cada vez más extendidos, tales como el estrés, el burn out, la violencia laboral, la fatiga crónica, los trastornos del sueño, las adicciones, etc., comenzaron a ser objeto de preocupación en el mundo del trabajo y estimularon la aparición de nuevas corrientes para analizar estas problemáticas.

El enfoque de los Riesgos Psicosociales en el Trabajo (RPST) constituye una perspectiva innovadora y ampliada, que se propone superar los enfoques tradicionales de la seguridad e higiene del trabajo. Primordialmente, permite detectar una serie de fenómenos -los riesgos psicosociales- que se generan en los entornos laborales y que son dificiles de aprehender por su naturaleza más intangible, pero que generan un daño real sobre la salud de los trabajadores. El punto de partida del enfoque de los RPST establece que la raíz última y principal de estos riesgos psicosociales reside en la forma en que se halla organizado el proceso de trabajo (Neffa, 2015; Gollac, 2011). En este sentido, si bien sus impactos son captados a través de las percepciones de los trabajadores -por lo cual estos tienen un papel protagónico en la detección de los RPST-, es al nivel del proceso de trabajo -de su organización y contenido- donde debe indagarse analíticamente e intervenirse para eliminarlos. De esta forma, este enfoque es una superación de las explicaciones individualizadoras sobre los padecimientos en el trabajo, que sitúan las causas de estos trastornos en el propio trabajador -ya sea por "problemas psíquicos o personales", o por una supuesta "incapacidad de adaptarse" a las exigencias laborales.

En el ámbito internacional, son una importante referencia los trabajos de Michel Gollac $(2011,2012)$ que en el contexto francés han planteado un estudio integral de los RPST. Gollac (2011:31) los define como "los riesgos para la salud física, psíquica, mental y social de los trabajadores, que son generados por las condiciones y medio ambiente de trabajo susceptibles de interactuar con el funcionamiento psíquico y mental, con impactos sobre la organización o empresa donde éstos se desempeñan". Gollac postula también que los diversos factores de riesgo psicosocial pueden agruparse en seis grandes ejes: i) la intensidad del trabajo y tiempo de trabajo; ii) las exigencias emocionales; iii) el grado 
De Prácticas y discursos

Universidad Nacional del Nordeste

Centro de Estudios Sociales

Año 7, Número 9, 2018, Marzo

ISSN 2250-6942
Los usos de la investigación-acción-participativa [IAP] para el estudio de los riesgos psicosociales en el trabajo. Reflexiones a partir de una experiencia con representantes sindicales y trabajadores

The uses of participatory action research in the study of psychosocial risks at work. Reflections from an experience with trade unionists and workers

y tipo de autonomía; iv) las relaciones sociales y relaciones de trabajo; v) Ios conflictos éticos y de valores; vi) el grado de seguridad y estabilidad de la situación de trabajo.

Como puede apreciarse en la clasificación anterior, los RPST están vinculados a diversas facetas de la situación de trabajo y es por eso que su estudio se nutre necesariamente de distintas disciplinas (la Economía del Trabajo, la Sociología del Trabajo, la Ergonomía, la Psicología, entre otras), así como de diversas corrientes teóricas que permiten comprender el complejo entramado entre trabajo y salud.

Esto último nos permite comprender por qué un estudio sobre riesgos psicosociales muchas veces presupone la realización de una investigación-acción-participativa. Entre otras, el enfoque de los RPST se nutre de una corriente analítica denominada Ergonomía de la actividad, que tiene como uno de sus principios rectores la diferencia entre trabajo prescripto y actividad (Wisner, 1998). En otras palabras, para poder cumplir con los objetivos asignados, los trabajadores hacen siempre muchas más cosas que las que les prescribieron. Al respecto, deben poner en tensión sus capacidades, usar su creatividad o astucia para hacer frente a errores, insuficiencias o fallas, para lo cual requiere el despliegue creativo de las capacidades mentales y el ejercicio de sus dimensiones afectivas y relacionales. También deben hacer frente a los incidentes o interrupciones imprevistas que ocurren durante el proceso productivo y obstaculizan el logro del objetivo asignado en el tiempo previsto (Neffa, 2015). De ahí que no sea suficiente con conocer el perfil de los puestos (descripción formal de las tareas prescriptas), sino que debe indagarse en profundidad qué es lo que los trabajadores realmente hacen y qué actividades despliegan cotidianamente.

Por eso, un estudio integral de los RPST requiere la participación de los trabajadores, porque ellos son los que mejor conocen su trabajo, los problemas que enfrentan y también quienes mejor pueden proponer soluciones y evaluar su efectividad.

La participación de los trabajadores y de sus representantes sindicales puede desarrollarse en diversas fases de una investigación de RPST: al momento de plantear la demanda y en la definición de los problemas que serán analizados; durante la recolección de datos, ofreciendo su percepción sobre las condiciones de trabajo y los problemas que enfrentan; por último, en la formulación de propuestas de intervención, en su implementación y evaluación. 
De Prácticas y discursos

Universidad Nacional del Nordeste

Centro de Estudios Sociales

Año 7, Número 9, 2018, Marzo

ISSN 2250-6942

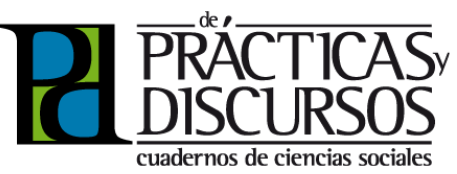

La investigación de RPST que reseñaremos en las próximas páginas constituye un ejemplo de este tipo de investigación participativa y podrá verse cómo en sus distintas etapas se desarrolló un trabajo común con los destinatarios.

\section{EL ESTUDIO DE LOS RPST EN UNA ORGANIZACIÓN PÚBLICA CON UNA METODOLOGÍA MIXTA}

En 2014, miembros del Secasfpi se acercaron al equipo interdisciplinario al que pertenecemos, dirigido por Julio C. Neffa, expresando la necesidad de un estudio científicamente avalado que refleje las condiciones de trabajo imperantes en la Anses. Los delegados habian tenido oportunidad de recorrer distintas dependencias en toda la Argentina y fueron encontrando que malestares y dolencias psicofísicas de diversa indole afectaban crecientemente a los trabajadores de la Anses. Pero debido a su complejidad y su naturaleza intangible, los RPST suelen permanecer invisibilizados y los empleadores frecuentemente se niegan a reconocer su existencia. De ahí la necesidad del sindicato de contar con un estudio objetivo y científico que avale lo que ellos estaban detectando y que les permita ulteriormente negociar una política preventiva integral.

En particular, se percibía desde el sindicato que los trabajadores en puestos de atención al público eran quienes manifestaban sentirse más "desbordados" por la gran afluencia de beneficiarios a las dependencias. Expresaban malestares tales como estrés, fatiga, y una diversidad de dolencias crónicas que identificaban como efecto de su trabajo. Fue por ello que, en términos metodológicos, la investigación se circunscribió desde el inicio a los trabajadores en puestos de atención al público, dado que era prioritario conocer su situación para encarar pronto medidas de intervención.

Esta franja de población trabajadora afectada se condice con las transformaciones organizacionales que ha tenido en el periodo reciente la Anses, especialmente desde 2003. El rol protagónico que adquirió esta institución como ejecutora de las -nuevas y numerosas- políticas sociales durante todo el periodo la llevó a un proceso continuo de ampliación de funciones. En sus inicios, este organismo estatal se encargaba principalmente de gestionar las jubilaciones y pensiones de los ciudadanos argentinos, pero paulatinamente fue incorporando la prestación de nume- 
De Prácticas y discursos

Universidad Nacional del Nordeste

Centro de Estudios Sociales

Año 7, Número 9, 2018, Marzo

ISSN 2250-6942
Los usos de la investigación-acción-participativa [IAP] para el estudio de los riesgos psicosociales en el trabajo. Reflexiones a partir de una experiencia con representantes sindicales y trabajadores

The uses of participatory action research in the study of psychosocial risks at work. Reflections from an experience with trade unionists and workers

rosos servicios: asignaciones familiares, becas para estudiantes, administración de créditos de vivienda, seguros de desempleo, préstamos para jubilados, pensiones por discapacidad, entre otros servicios sociales. Así, en 2016, desde la Anses se administraban 17 millones de beneficios sociales mensuales (Anses, 2016) y el número puede seguir en aumento. Internamente, esto ha implicado la adición permanente de nuevas tareas, cambios en el contenido del trabajo, introducción de tecnologías, mayor demanda de sus servicios, entre otras cuestiones.

La investigación RPST se desarrolló en 2015 y 2016, buscando abarcar las diversas realidades laborales existentes en la Anses. De esta forma, el estudio tuvo una cobertura geográfica muy extensa, dado que se relevaron datos en todas las regionales del país, lo cual implicó un esfuerzo muy grande de planificación, organización y logística. Asimismo, la Anses constituye una organización con una cuantiosa dotación de trabajadores, los cuales se estiman en 16000 aproximadamente, distribuidos en más de 300 dependencias en toda la Argentina.

Esta breve descripción sobre la Anses y el tipo de actividades que allí se desarrollan servirán para comprender mejor los rasgos de la investigación emprendida y los desafíos a los cuales buscó responder.

\section{ANÁLISIS Y DISCUSIÓN DE ALGUNAS DIMENSIONES SIGNIFICATIVAS DE LA IAP REALIZADA}

El desarrollo de una IAP implica muchas fases y aspectos donde es fructifero reflexionar sobre las dinámicas establecidas entre los diversos actores involucrados -investigadores, representantes del sindicato, delegados, trabajadores y otros- para reseñar qué papel jugó cada uno en el desarrollo de la investigación y cómo se llegaron a concretar las metas planteadas. En este artículo nos centraremos en tres aspectos, que desarrollaremos a continuación: la composición del grupo de trabajo, el desarrollo del trabajo de campo y la elaboración del diagnóstico y de las propuestas de intervención.

\subsection{COMPOSICIÓN Y CONFORMACIÓN DEL EQUIPO DE TRABAJO}

Una tarea inicial en este tipo de investigaciones, que reviste gran importancia, es la conformación del Grupo Responsable, el cual necesariamente debe estar conformado por miembros de las 
De Prácticas y discursos

Universidad Nacional del Nordeste

Centro de Estudios Sociales

Año 7, Número 9, 2018, Marzo

ISSN 2250-6942

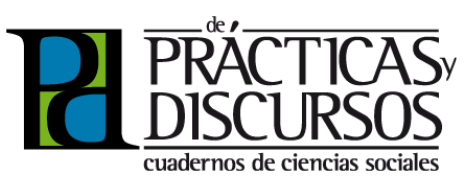

dos partes: por un lado, los investigadores sociales y, por otro, aquellos miembros del grupo destinatario, los que intervendrán activamente en la programación y ejecución de todo el proyecto. En lo referente a los investigadores sociales, el equipo conformado para la investigación en la Anses se caracterizó por su pertenencia a distintas disciplinas. Los profesionales fueron convocados en virtud de su capacidad teórica-metodológica para abordar la cuestión de los RPST desde su campo de pertenencia y por su experiencia previa en programas de esta naturaleza, lo que facilitó su inserción en una dinámica de trabajo participativa. Sus enfoques se complementaron y permitieron echar luz sobre distintas facetas de la situación de trabajo en la Anses, que luego se integraron en un análisis general al momento de elaborar el diagnóstico de base.

Específicamente, el equipo de investigadores estuvo conformado por los siguientes profesionales:

1. Una psicóloga del trabajo, quien estuvo a cargo de desarrollar Talleres de Visualización con los trabajadores. Estos talleres - de 8 horas de duración- fueron organizados en distintas partes del país y permitieron que los trabajadores pudieran expresar vivencias, preocupaciones, padecimientos, recomendaciones, etc., en relación a su trabajo. Asimismo, esta profesional luego coordinó Talleres de Devolución en la fase final del proyecto, donde también se convocó a los trabajadores para hacerles una devolución de los resultados encontrados y poner a discusión las propuestas de intervención formuladas.

2. Un ergónomo, encargado de realizar relevamientos en dependencias de la Anses. El objetivo fue conocer las características de los puestos y las Condiciones y Medio Ambiente de Trabajo (CYMAT) en dependencias seleccionadas de distintas ciudades. Este profesional realizó mediciones objetivas, pero también se encargó de conversar con los trabajadores in situ sobre diferentes cuestiones relacionadas a su proceso de trabajo, los problemas percibidos y las condiciones generales de cada centro laboral.

3. Un médico del trabajo, encargado de buscar y sistematizar la información relativa al servicio de medicina laboral vigente en la organización, así como el estado general de salud de los trabajadores, detectando patologías frecuentes.

4. Dos sociólogas, encargadas de coordinar el diseño, la aplicación y el análisis de la encuesta de RPST, la cual buscó relevar 
De Prácticas y discursos

Universidad Nacional del Nordeste

Centro de Estudios Sociales

Año 7, Número 9, 2018, Marzo

ISSN 2250-6942
Los usos de la investigación-acción-participativa [IAP] para el estudio de los riesgos psicosociales en el trabajo. Reflexiones a partir de una experiencia con representantes sindicales y trabajadores

The uses of participatory action research in the study of psychosocial risks at work. Reflections from an experience with trade unionists and workers

la percepción de los trabajadores sobre distintos factores de riesgo psicosocial.

5. Por su parte, la dirección del proyecto estuvo a cargo de un economista del trabajo que también pudo proveer su impronta disciplinar al proyecto.

Esta interdisciplinariedad del equipo de investigadores y un abordaje metodológico mixto -combinación de técnicas cualitativas y cuantitativas de recolección de información- permitieron obtener una mayor comprensión del problema bajo estudio, partiendo del hecho de que la relación entre trabajo y salud constituye un entramado complejo que no se agota en un solo enfoque y que tiene múltiples planos (Hugentobler, Israel y Schurman, 1992).

Luego, por la parte del sindicato, se contó con la participación de muchisimas personas, pero fue fundamental la participación activa de tres de sus miembros: el secretario adjunto, la secretaria de Cultura y Capacitación, y la representante en la Comisión de CyMAT. A modo de coordinadores, ellos pusieron a disposición toda la estructura de delegados para colaborar con la investigación. Particularmente, estos tres representantes se involucraron con la investigación en sus diferentes fases:

-Ayudando a definir la situación-problema: explicitando las necesidades y los intereses de la organización sindical y de los trabajadores, así como los aspectos sobre los cuales se esperaba intervenir para encontrar soluciones.

-Participando del diseño: al inicio y durante las etapas subsecuentes, los representantes del Secasfpi brindaron valiosísima información que ayudó al equipo de investigadores a proponer un diseño de investigación consensuado, viable en términos operativos y con las técnicas más adaptadas al campo bajo estudio.

-Colaborando en el trabajo de campo: acompañando personalmente y a través de sus delegados el desarrollo del trabajo de campo, indicando los lugares y momentos adecuados para su realización, brindando recursos y apoyo logístico, entre otros.

De esta forma, pudo generarse un genuino intercambio entre ambas partes, donde los conocimientos del equipo de investigadores se vieron complementados y enriquecidos por los saberes que aportaron los miembros del Secasfpi. Ellos mostraron un verdadero compromiso con las tareas a realizar, brindando su tiempo y todos los recursos a su alcance para alcanzar los objetivos comunes planificados.

De igual forma, mostraron una gran motivación para conocer los 
De Prácticas y discursos

Universidad Nacional del Nordeste

Centro de Estudios Sociales

Año 7, Número 9, 2018, Marzo

ISSN 2250-6942

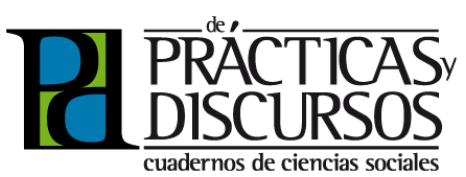

aspectos metodológicos e incluso conceptuales que subyacen a un estudio de RPST. En este punto, el equipo de investigadores trató de responder a todas sus inquietudes y de facilitarles instancias de diálogo permanente. Paulatinamente, esto les permitió a los miembros del Secasfpi apropiarse de la temática y situarlos en mejores condiciones para ser parte activa del proceso de toma de decisiones que requirió la investigación.

\subsection{DESARROLLO DEL TRABAJO DE CAMPO: EL CASO DE LA ENCUESTA}

En toda investigación, el desarrollo del trabajo de campo constituye un aspecto de gran importancia y que requiere de distintas acciones que la faciliten. Al respecto, una de las acciones iniciales que emprendió el Grupo Responsable fue informar sobre el proyecto a los miembros de la propia organización sindical e invitarlos a que se implicaran activamente. Primero, se enviaron cartas con esta información y luego, se organizó una reunión presencial en la ciudad de Buenos Aires, previa al inicio del trabajo de campo, a la que concurrieron delegados regionales provenientes de todo el país.

En esta reunión se produjo un rico intercambio, donde los delegados pudieron interiorizarse en detalle sobre el proyecto y en la que también emitieron sugerencias. Un punto que resultó importante fue debatir por qué esta investigación era innovadora y en qué se diferenciaba de otras iniciativas en las cuales ya habian participado previamente, más orientadas a temas de seguridad e higiene. Suele suceder que esas experiencias previas dejan su huella en estos actores, demarcando modos de accionar esperados e incluso horizontes de expectativas determinados, más aun cuando muchas de las iniciativas en las cuales han participado, han tenido una eficacia limitada en sus intervenciones. Al respecto, fue importante integrar a los delegados en la discusión sobre los riesgos psicosociales y en qué sentido este enfoque podia ser más atinente para encarar los problemas de salud de los trabajadores en la Anses.

Después de esa reunión inicial, los delegados regresaron a sus localidades de origen y se encargaron de difundir la información a distintos actores que -directa o indirectamente- iban a estar involucrados en la investigación: otros delegados del Secasfpi, delegados de los otros sindicatos, autoridades de las dependen- 
De Prácticas y discursos

Universidad Nacional del Nordeste

Centro de Estudios Sociales

Año 7, Número 9, 2018, Marzo

ISSN 2250-6942
Los usos de la investigaciōn-acción-participativa [IAP] para el estudio de los riesgos psicosociales en el trabajo. Reflexiones a partir de una experiencia con representantes sindicales y trabajadores

The uses of participatory action research in the study of psychosocial risks at work. Reflections from an experience with trade unionists and workers
${ }^{6}$ Para conocer más detalles sobre la metodología específica de la encuesta, se sugiere ver Henry (2016).

${ }^{7}$ Esto se hizo para evitar que los trabajadores y los delegados supieran con anticipación quiénes efectivamente iban a ser encuestados. De esta forma, se impedía una inducción previa de respuestas por influencia del entorno social. cias y, primordialmente, los propios trabajadores de la Anses. Esto último era considerado fundamental para favorecer el porcentaje de respuesta voluntaria a la encuesta y para motivarlos a participar de los talleres.

El caso de la encuesta resulta muy pertinente para ilustrar cómo se realizó el trabajo mancomunado entre las partes involucradas en la investigación y por eso quisiéramos detenernos a reseñar sus puntos más salientes. En cuanto a sus características, cabe señalar que se recogieron 694 encuestas en dependencias de la Anses de todo el pais. La misma tuvo formato en papel, fue administrada por encuestadores capacitados para tal fin, de forma presencial y su duración promedio fue de 40 a 50 minutos. Se trató de una participación voluntaria, anónima, y en todos los casos los encuestadores primero ofrecieron información a los trabajadores sobre las características del estudio, su temática, su confidencialidad y las instituciones intervinientes. Asimismo, es importante remarcar que la encuesta fue respondida por trabajadores de distintos perfiles y que no era necesario que sean afiliados al Secasfpi. Por el contrario, se trató de un relevamiento abierto a todos los trabajadores y con vocación de generar resultados que representen a todo el colectivo ${ }^{6}$.

Como puede vislumbrarse, el trabajo de campo de la encuesta representó un gran desafío por la enorme extensión geográfica que debía abarcar. Al respecto, fue fundamental la ayuda de los miembros del sindicato para programar los "circuitos" de encuesta y la logística necesaria. Y si bien estos circuitos fueron armados en función de abarcar localidades cercanas, esto no siempre fue posible dadas las dimensiones de nuestro país. Así, hubo casos donde los encuestadores tuvieron que viajar más de 500 km entre una dependencia de la Anses y otra para relevar un mismo circuito regional.

En función de esta planificación por circuitos, el Equipo Responsable fue contactando a los delegados de las distintas regionales para que tomasen parte del operativo de encuesta en tanto "facilitadores" locales. En el momento de su llegada a la dependencia, y no antes, el encuestador informaba al delegado y/o al responsable de la dependencia a qué trabajadores deseaba encuestar y cuyos nombres estaban indicados en el listado de la muestra ${ }^{7}$. Seguidamente, el delegado -con ayuda del representante de la dependencia- se encargaba de ubicar a los trabajadores requeridos y de conseguir un lugar tranquilo, con privacidad para realizar la entrevista. 
De Prácticas y discursos

Universidad Nacional del Nordeste

Centro de Estudios Sociales

Año 7, Número 9, 2018, Marzo

ISSN 2250-6942

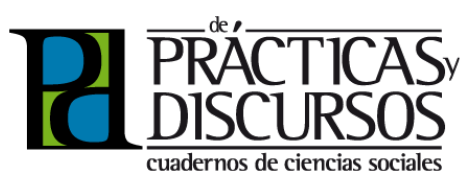

Así, los delegados del Secasfpi tuvieron una labor de acompañamiento y de colaboración permanente con los equipos de encuestadores. En primer término, les facilitaron todas las cuestiones logísticas y operativas requeridas: traslados, arreglos de alojamiento, etc. En segundo término, fueron agentes de orientación para los encuestadores, ya que pudieron proveerles de información suplementaria sobre cada uno de los lugares a encuestar (horarios más favorables, circulación por los edificios, datos de interés, etc). Esto resultaba muy valioso para que los encuestadores pudieran delinear la estrategia de entrada al campo y pudieran obtener -en corto tiempo- un panorama general sobre la dependencia a la que llegaban.

Mientras tanto, las dos coordinadoras de campo localizadas en la ciudad de Buenos Aires y La Plata supervisaron en todo momento la tarea de los encuestadores en distintas partes del pais. En este proceso, estuvieron en todo momento acompañadas por los representantes del Secasfipi designados para colaborar con la investigación, también localizados en Buenos Aires, en la sede central del sindicato. Ellos, a su vez, coordinaban acciones con los delegados y favorecian la articulación local con los encuestadores que estaban recorriendo el país. De esta forma, se estableció una estructura operativa mixta y gracias a esta estrecha colaboración, la encuesta tuvo un desarrollo expeditivo -se completó en cuatro meses-, con mínimos contratiempos a lo largo de todo el proyecto.

\subsection{LA ELABORACIÓN DEL DIAGNÓSTICO Y DE LAS PROPUESTAS DE INTERVENCIÓN}

Luego de efectuados los relevamientos correspondientes a las cuatro técnicas (talleres, encuesta, estudio de ergonomía y de medicina del trabajo), se ingresó a la fase de análisis de los datos. Durante la misma, los integrantes del equipo de investigación se encargaron de acopiar la información y de articularla en pos de generar un primer documento donde se exponga el diagnóstico integral sobre los RPST en la Anses. Asimismo, y como tarea central, se elaboró una primera serie de propuestas concretas de intervención sobre los problemas detectados.

A partir de este trabajo de integración de contenidos, el equipo de investigadores realizó una versión preliminar del Informe Final que tuvo sucesivos momentos de revisión por parte del sindicato. En primer lugar, se realizó un ciclo de reuniones inter- 
De Prácticas y discursos

Universidad Nacional del Nordeste

Centro de Estudios Sociales

Año 7, Número 9, 2018, Marzo

ISSN 2250-6942
Los usos de la investigación-acciôn-participativa (IAP] para el estudio de los riesgos psicosociales en el trabajo. Reflexiones a partir de una experiencia con representantes sindicales y trabajadores

The uses of participatory action research in the study of psychosocial risks at work. Reflections from an experience with trade unionists and workers

nas con los representantes del Secasfpi que formaban parte del Equipo Responsable, durante las cuales se puso a discusión el documento, para cotejar interpretaciones y recibir sugerencias. En función de estos encuentros, se hicieron varios ajustes y se modificaron cuestiones que los investigadores quizás no habian abordado adecuadamente y se enriquecieron algunas facetas a partir de datos y propuestas que no se habian contemplado. Posteriormente, una versión de este Informe Preliminar fue presentada y discutida durante una jornada de trabajo en la ciudad de Buenos Aires, a la cual se invitó a dirigentes y delegados provenientes de diversas regionales del pais, (que habian recibido el documento con anticipación para su cuidadosa lectura). En dicho encuentro, se les solicitó que emitieran sus comentarios y propuestas previos a la redacción definitiva del Informe Final. De igual forma, se les pidió que evaluaran la viabilidad de las recomendaciones formuladas, las prioridades entre ellas y que elaboraran las estrategias para ponerlas en práctica. Una vez más, el debate fue muy importante porque permitió realizar nuevos ajustes al diagnóstico y a las propuestas de intervención. Por último, el diagnóstico y las propuestas del Informe fueron puestos a discusión en los Talleres de Devolución, con trabajadores de la Anses en distintas partes del país.

Todas estas instancias sucesivas buscaron involucrar genuinamente a los delegados y a los trabajadores para que puedan apropiarse del conocimiento generado y retroalimentarlo desde sus perspectivas. Como fruto de este trabajo colectivo, pudo elaborarse un Informe Final de la investigación muy completo, donde la rigurosidad metodológica fue acompañada de los valiosos aportes de los destinarios, interesados en producir cambios sobre su situación. Al respecto, en el Informe se expuso de manera detallada un conjunto de riesgos psicosociales detectados en la Anses y que aquí mencionaremos sintéticamente por cuestiones de espacio: alta intensidad en los ritmos de trabajo; importante incidencia de la carga mental; fuertes exigencias emocionales que sufren los trabajadores por el contacto directo con personas en situación de sufrimiento y vulnerabilidad social; situaciones de violencia verbal y de agresiones en los puestos de atención al público; falta de capacitación y de formación para la resolución de las tareas que provoca en los trabajadores una autonomía forzada y padecida; falta de reconocimiento por parte de la organización (ausencia de evaluaciones y de posibilidades de carrera); una insuficiencia 
De Prácticas y discursos

Universidad Nacional del Nordeste

Centro de Estudios Sociales

Año 7, Número 9, 2018, Marzo

ISSN 2250-6942

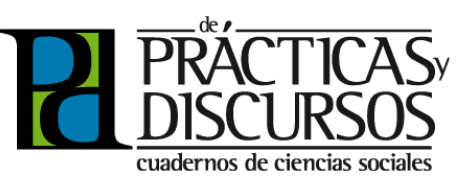

${ }^{8}$ Para conocer el diagnóstico completo de los riesgos psicosociales detectados y las propuestas de intervención elaboradas, se sugiere consultar Neffa, Korinfeld y Henry (2017). en actividades de prevención y de formación en riesgos del trabajo; entre otros aspectos ${ }^{8}$.

Al momento de escribir estas páginas, la investigación se encuentra en fase de difusión, dada la importancia de seguir comunicando los resultados y propuestas entre todos los potenciales beneficiarios. Esta tarea se ha encarado de diversas maneras: en primer lugar, el Informe Final fue editado bajo el formato de libro (Neffa, Korinfeld y Henry, 2017) con apoyo del sindicato como manera de cristalizar el trabajo realizado y de hacerlo accesible a un amplio público. Segundo, la investigación y el libro en particular están siendo presentados en variadísimos espacios: conferencias, encuentros intersindicales, talleres con trabajadores, ferias del libro, congresos académicos, cursos universitarios, etc. De igual forma, el libro está siendo distribuido gratuitamente a todos los delegados del país, para que lo socialicen en sus centros de trabajo con sus compañeros.

Al respecto, cabe señalar que tanto en la confección del Informe Final como del libro se procuró utilizar un lenguaje claro y accesible para que su lectura resulte sencilla a delegados y trabajadores. En una IAP, la comunicabilidad de los resultados es un aspecto clave que no debe descuidarse si se desea transferir efectivamente los conocimientos a la comunidad y posibilitar su más amplia difusión.

Actualmente, el sindicato se encuentra haciendo gestiones para poder elevar formalmente los resultados de la investigación a las autoridades de la Anses y presentarles la situación detectada. En este punto, el sindicato quisiera proponer y debatir, en una mesa de trabajo conjunta, el desarrollo de una política integral y de largo plazo que apunte a prevenir los riesgos psicosociales en la Anses. Es fundamental que esta discusión se realice de manera participativa y que luego, en el marco de un comité mixto, se monitoree su aplicación y se haga un seguimiento cotidiano. Se considera que los conocimientos adquiridos durante la investigación han provisto de herramientas y de nuevos saberes a los miembros del Secasfpi, por lo que podrán cumplir esta labor con mucha eficacia y autonomía en el futuro.

\section{CONCLUSIONES}

En este artículo hemos podido presentar un caso de investigación-acción-participativa, con el objetivo de reseñar sus aspectos más destacados y mostrar la forma concreta en que puede 
De Prácticas y discursos

Universidad Nacional del Nordeste

Centro de Estudios Sociales

Año 7, Número 9, 2018, Marzo

ISSN 2250-6942
Los usos de la investigación-acciôn-participativa (IAP] para el estudio de los riesgos psicosociales en el trabajo. Reflexiones a partir de una experiencia con representantes sindicales y trabajadores

The uses of participatory action research in the study of psychosocial risks at work. Reflections from an experience with trade unionists and workers

articularse el trabajo entre investigadores y un grupo social, en este caso un sindicato, para generar conocimiento e intervenir sobre una situación-problema que los aqueja.

En primer término, hemos explicado los rasgos que adoptó el Equipo Responsable y su conformación mixta. Por el lado de los investigadores, se procuró que hubiese una multiplicidad de miradas para abordar el problema bajo estudio $y$, en ese sentido, fue muy enriquecedor contar con profesionales de distintas disciplinas y que ya habian realizado experiencias de este tipo, por lo cual estaban sensibilizados en estas investigaciones participativas. Por el lado del sindicato, fue vital contar con miembros muy activos y comprometidos con el proyecto. Su participación en todas las fases otorgó al estudio una viabilidad muy grande y permitió que se tomaran en conjunto las muchísimas decisiones que iban surgiendo en el camino.

En segundo término, expusimos las características que adoptó el trabajo de campo referido a la encuesta nacional de RPST y las acciones conjuntas desarrolladas en esta instancia. Particularmente, la encuesta fue aplicada en una extensión territorial muy grande, involucrando a numerosos actores y en un tiempo relativamente breve, por lo que el apoyo brindando por el Secasfpi resultó fundamental en el alcance de las metas propuestas. De manera coordinada se fueron visitando los diferentes circuitos y se fue consolidando una forma de trabajo donde el desempeño de cada uno de los actores -tanto los localizados en Buenos Aires como en distintas partes del país- cumplió un rol fundamental y permitió el trabajo de los otros durante la encuesta.

Por último, hemos reseñado cómo se realizó la formulación del diagnóstico y de las propuestas de intervención sobre los problemas detectados. Se elaboraron versiones sucesivas que fueron sometidas a discusión, análisis y crítica por parte de los delegados del Secasfpi. Asimismo, los contenidos de este informe fueron debatidos con los trabajadores en los Talleres de Devolución. En todos los casos, su conocimiento situado y la experiencia acumulada fueron insumos clave que permitieron alcanzar un diagnóstico integral y una serie de propuestas de intervención que sean realistas y que contemplen la perspectiva de los propios trabajadores.

En suma, esta experiencia de colaboración entre academia y ámbito sindical nos lleva a afirmar el valor que todavía conservan algunos de los principios del Modelo Obrero: la no delegación de la salud, la participación activa de los propios trabaja- 
dores y sus representantes, y la recuperación de sus saberes en la formulación de diagnósticos y propuestas. Asimismo, confiamos en que los aspectos expuestos en este artículo sirvan como aliciente para que se produzcan más estudios con esta orientación y se siga nutriendo así una larga tradición en el campo de la salud laboral que a veces queda olvidada en los ámbitos hegemónicos, pero que conserva toda su vigencia y su potencial en tanto existan condiciones perjudiciales para los trabajadores que deban ser transformadas.

\section{BIBLIOGRAFÍA}

ANDER-EGG, E. (2002). Repensando la Investigación-AcciónParticipativa. Buenos Aires: Lumen-Hvmanitas.

ANSES (2016). Más de 16 millones de asignaciones, jubilaciones y pensiones actualizadas, Revista Argentinos y la Seguridad Social, año 5, 23, 22-25. Disponible en: http://www.anses.gob.ar/ archivos/publicacion/RevistaArgentinos-23.pdf

BALCAZAR, F.E. (2003). Investigación acción participativa (IAP): Aspectos conceptuales y dificultades de implementación. Fundamentos en Humanidades, año IV, I/II, 59-77.

CAMPOS, A. (1990). Investigación participativa: reflexiones acerca de sus fundamentos metodológicos y de sus aportes al desarrollo social. Cuadernos de Desarrollo Rural, 24, 129-146.

GOLLAC, M. (2011) (coord). Mesurer les facteurs psychosociaux de risque au travail pour les maitriser. Paris: Rapport au ministre du Travail, de l'Emploi et de la Santé.

GOLLAC, M. (2012). Les risques psychosociaux au travail : d'une "question de société» à des questions scientifiques. Introduction. Travail et emploi, 129, 5-10.

HENRY, M.L. (2016). La encuesta como herramienta de recolección de datos sobre riesgos psicosociales. Características y balance metodológico del relevamiento realizado entre trabajadores de la ANSES. Ponencia presentada en las IX Jornadas de Sociología de la UNLP. La Plata, 5-7 de diciembre. 
De Prácticas y discursos

Universidad Nacional del Nordeste

Centro de Estudios Sociales

Año 7, Número 9, 2018, Marzo

ISSN 2250-6942
Los usos de la investigación-acción-participativa [IAP] para el estudio de los riesgos psicosociales en el trabajo. Reflexiones a partir de una experiencia con representantes sindicales y trabajadores

The uses of participatory action research in the study of psychosocial risks at work. Reflections from an experience with trade unionists and workers

HUGENTOBLER, M.K.; ISRAEL, B.A. Y SCHURMAN, S.J. (1992). An Action Research Approach to Workplace Health: Integrating Methods. Health Education \& Behavior, 19(1), 55-76.

LAURELL, C.A. (1984). Ciencia y experiencia obrera: la lucha por la salud en Italia. Cuadernos Políticos, 41, 63-83.

MARTíNEZ ALCÁNTARA, S. (2007). La investigación participativa como práctica social y su aportación al mundo laboral a través del Modelo Obrero. Salud de los Trabajadores, 2(15), 107-117.

NEFFA, J.C. (2015). Los riesgos psicosociales en el trabajo: contribución a su estudio. Caba: Ceil del Conicet; Corrientes: Unne, Fac. de Cs. Económicas; La Plata: UNLP, Fac. de Cs. Económicas; Moreno: Universidad Nac. de Moreno; Caba: Umet-Citra.

NEFFA, J.C.; KORINFELD, S. Y HENRY, M.L. (2017). Trabajo y salud en puestos de atención al público: una investigación sobre riesgos psicosociales en el trabajo en ANSES. Buenos Aires: Secasfpi.

PARK, P. (1992). Qué es la investigación-acción participativa: Perspectivas teóricas y metodológicas. En Salazar, M.C. (coord.) La investigación-acción participativa: inicios y desarrollos. Madrid: Editorial Popular: Organización de Estados Iberoamericanos para la Educación, la Ciencia y la Cultura, OEl: Sociedad Estatal Quinto Centenario.

VOGEL, L. (2016). La actualidad del modelo obrero italiano para la lucha a favor de la salud en el trabajo. Laboreal, 2(XII), 10-17.

WISNER, A. (1998). Ergonomía y condiciones de trabajo. Buenos Aires: Ceil/Conicet- Humanitas. 